\title{
Photo-Production of Proton Antiproton Resonances
}

\author{
Paul Eugenio and Burnham Stokes \\ for the CLAS Collaboration \\ Department of Physics, Florida State University, Tallahassee, FL, USA
}

\begin{abstract}
Preliminary results are reported on the reaction $\gamma p \rightarrow p p \bar{p}$. The data were obtained at the Thomas Jefferson National Accelerator Facility utilizing the CLAS detector and a tagged photon beam of 4.8 to $5.2 \mathrm{GeV}$ incident on a liquid hydrogen target. The focus of this study is to search for possible intermediate resonances which decay to $\bar{p} p$. Both final state protons were detected in CLAS whereas the antiproton was identified via missing mass. General features of the accepted data are presented.
\end{abstract}

\section{Introduction}

The proton-antiproton system has had a rich history spanning more than thirty years. Initially, the $p \bar{p}$ system had much interest due to theoretical predictions of exotic matter. These predictions included: nucleon-antinucleon states that are loosely bound in a molecule-like structure called quasi-nuclear baryonium, and tighly-bound multi-quark baryonium $(q q-\bar{q} \bar{q})$ which have favored decays to nucleon-antinucleon final states.

Arround 1970, there were claims of a unusually-narrow meson resonance with a mass of $1.93 \mathrm{GeV} / \mathrm{c}^{2}$ [1][2], and it was believed that this particle was not an ordinary meson and that it would couple to the proton-antiproton system. There were then claims that experiments found the narrow resonance in proton-antiproton scattering experiments [3][4][5][6]. Also, in the late 1970s there were claims of additional higher mass narrow resonances at 2.02 and $2.20 \mathrm{GeV} / \mathrm{c}^{2}$ in the proton-antiproton system [7][6][8]. However follow up experiments did not make such claims[9][10]. And until recently, the debate had died out.

In 1997, CERN refuted their own earlier claims of the 1.93 and $2.02 \mathrm{GeV} / \mathrm{c}^{2}$ resonances. Yet in 1999, a reanalysis of the CERN data confirmed the existence of the 2.02 and $2.2 \mathrm{GeV} / \mathrm{c}^{2}$ resonances. Presently, the only well-known particle that decays to proton-antiproton is the $J / \psi$ particle, with a mass of $3.097 \mathrm{GeV} / \mathrm{c}^{2}$ [11]. Most of the past experiments involved proton-antiproton scattering or pion production. Recently Jefferson Laboratory has provided the first look at the proton-antiproton system through photoproduction.

In 1999 JLAB observed approximately 2000 accepted events of photoproduction of proton-antiproton $(\gamma p \rightarrow p p \bar{p})$. This data was particularly exciting due to the fact that the yield was an order of magnitude larger than any previous photoproduction data set. Yet this data was still rather limited in statistics and preliminary studies of the protonantiproton system were inconclusive. The experiment which followed, JLAB E01-017, 
produced an order of magnitude greater number of $p \bar{p}$ events, and it is this data that is the main focus of the present work. With such a data set, it should be possible for the first time to search for the existence of both narrow and broad resonances via the use of partial wave analysis of the proton-antiproton system.

\section{Experimental Results}

The data were obtained using a photon beam incident on a liquid hydrogen target in the CEBAF Large Angle Spectrometer (CLAS). The particle detection system consists

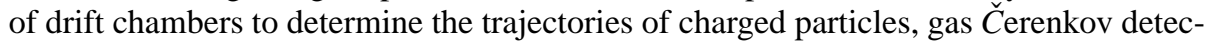
tors for particle identification, scintillation counters for measuring time-of-flight (TOF) and particle identification, and electromagnetic calorimeters to detect neutral particles [12]. These detectors are designed to provide as much coverage of the $4 \pi$ solid angle as possible.

For this experiment, the trigger required that a photon of an energy between 4.8 and $5.5 \mathrm{GeV}$ be detected, it required that at least two of the timing counters surrounding the target measure hits, and it required that at least two of the six downstream TOF sectors measure hits.

After filtering events by particle identification, initial cuts were applied to the data set. These selections include beam energy, vertex position, and timing requirements. The photon energy was determined using the electron beam tagger. While the trigger required that a photon with an energy in the range of 4.8 to $5.5 \mathrm{GeV}$ be identified, additional low energy photons could also be measured during the time window allowed to acquire the event. This would lead to an ambiguity in which photon beam particle was associated with the event measured in CLAS. Tight timing requirements as well as energy conservation cuts were used to take this ambiguity into account. Events were required to have a beam energy in the range of 4.8 to $5.5 \mathrm{GeV}$, and events below this energy are excluded from further analysis.

Nearly five thousand exclusive events were observed where all final state particles were identified in the CLAS spectrometer. However in CLAS, there are detector regions where particles can go unmeasured. For example, the CLAS toroidal magnetic field bends negatively charged particles back toward the beam. Quite often, these particles end up going back into the beam-line, and are lost. To increase the exclusive data yield, the anti-proton was allowed to be indetified via the missing mass.

Figure 1 shows the missing-mass-squared of events containing two identified protons. There is a prominent peak at a mass squared of $0.880\left(\mathrm{GeV} / \mathrm{c}^{2}\right)^{2}$, which is consistent with a missing antiproton. Selecting the events consistent with a missing antiproton $\left(0.85\left(\mathrm{GeV} / \mathrm{c}^{2}\right)^{2} \leq M M^{2} \leq 0.91\left(\mathrm{GeV} / \mathrm{c}^{2}\right)^{2}\right)$ yields approximately $17,100 \gamma p \rightarrow p p(\bar{p})$ events. Yet not all of these events are $\gamma p \rightarrow p p \bar{p}$ events as seen by the nearly flat background of non-antiprotons in the missing-mass squared distribution. Efforts are underway to further clean up and understand the background events under the antiproton signal.

Possible production mechanisms which describe the photoproduction of a protonantiproton pair are diffraction/meson exchange, baryon exchange, and antibaryon ex- 


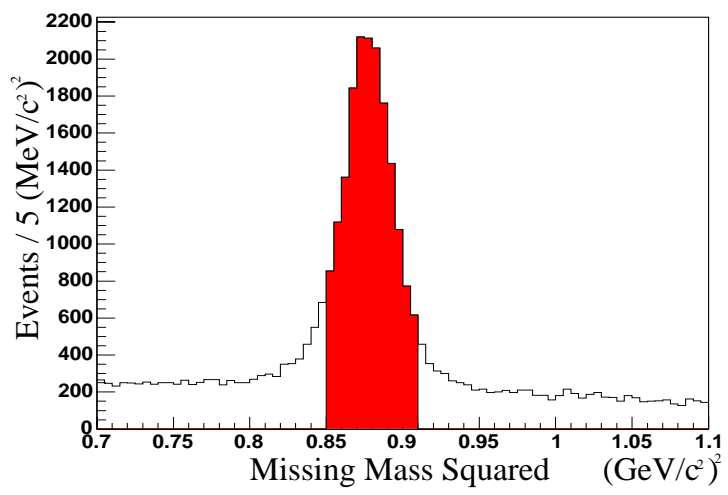

FIGURE 1. Missing Mass Squared off two protons.

change. In each process, an intermediate resonance may be produced. In meson exchange the photon transfers very little momentum to the target, but interacts, causing the photon to produce a resonance that decays to a fast forward-going proton-antiproton pair. In baryon exchange, the photon interacts with an exchange baryon converting it to a fast forward-going proton leaving behind a slow moving resonance at the target vertex which decays to a proton-antiproton pair. For antibaryon exchange, the photon interacts with an exchange antibaryon converting it to a fast forward-going antiproton, leaving behind a resonance at the target vertex which decays to two protons.

The distinction of meson exchange and baryon exchange production is clouded by the two identical protons. Without information identifying which is which, the two mechanisms are nearly indistinguishable. For antibaryon exchange it does not matter since both protons are at the same decay vertex. The two proton invariant mass is shown in Fig. 2. No obvious peaks or features are observed.

A simple way to distinguish the protons is by storting on the proton momentum. In the cases of meson and baryon exchange, one proton should be moving fast and in the forward direction. Whereas the other proton being produced at or near the target vertex receives very little momentum transfer from the beam and is expected to be slow going. Therefore, one can use the momentum of the two protons on an event by event basis and associate a $p_{\text {fast }} \bar{p}$ resonance with meson exchange and a $p_{\text {slow }} \bar{p}$ resonance with baryon exchange.

In Fig. 3, the proton momentum distribution is shown. The proton with the greatest magnitude of momentum is defined as the fast proton, and the other proton is defined as the slow proton. The light histogram shows the event by event distribution for the slow protons whereas the dark histogram is that for the fast protons. In addition, there are other kinematic variables that may help distinguish production mechanisms. These observables are currently under investigation.

The invariant mass of $p_{\text {slow }} \bar{p}$ is shown in Fig. 4. The distribution has some interesting structures, with a sharp rise at threshold and a possible narrow peak or dip near $2.0 \mathrm{GeV}$ 


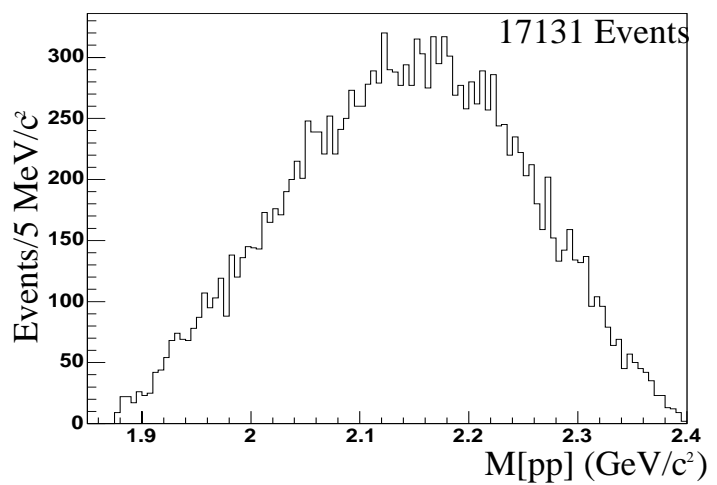

FIGURE 2. Accepted invariant mass distribution of the two protons.

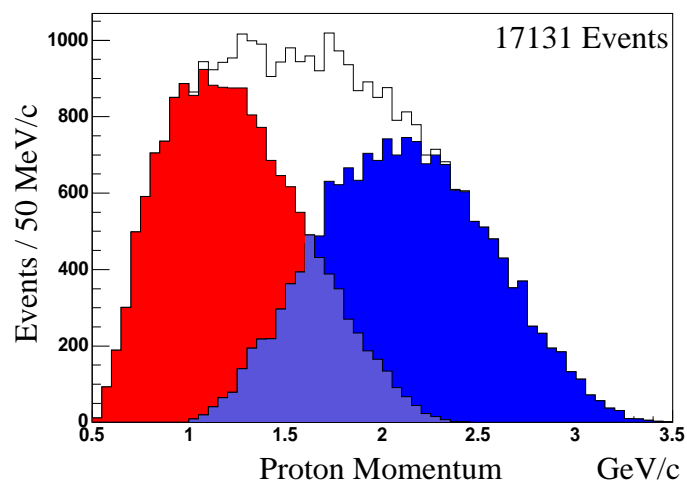

FIGURE 3. Proton momentum distributions. In each event, whichever proton has less momentum is placed in the "slow" distribution (left) and the other goes into the "fast" distribution (right)

and broader peak at $2.04 \mathrm{GeV}$. In the invariant mass distribution of $p_{\text {fast }} \bar{p}$ (Fig. 5 ) there are no obvious structures.

Future analysis plans include understanding background events, exploring kinematical features of the data, analyzing angular distributions, performing Monte Carlo simulations, and performing a partial wave analysis to search for resonant behavior.

\section{REFERENCES}

1. M.N. Focacci et. al., Phys. Rev. Lett. 17, 890(1966).

2. D. Cline et. al., Phys. Rev. Lett. 17, 1268(1968). 


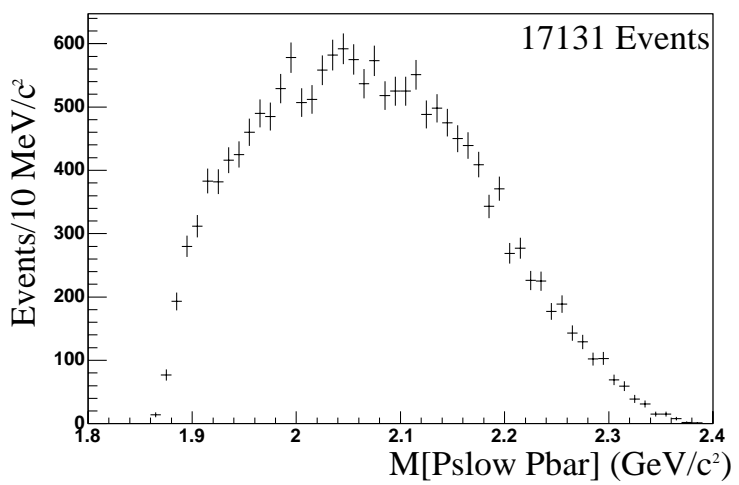

FIGURE 4. The invariant mass of the slow proton with the antiproton.

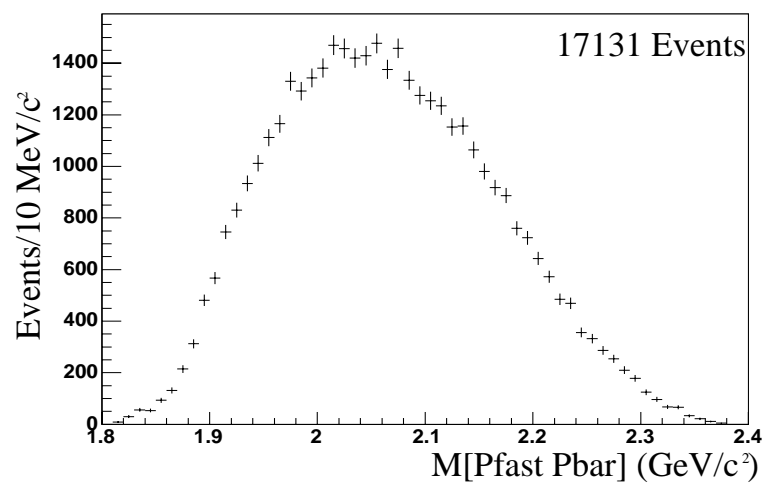

FIGURE 5. The invariant mass of the fast proton with the antiproton.

3. A.S. Carroll et. al., Phys. Rev. Lett. 32, 247(1974).

4. T.E. Kalogeropoulos and G.S. Tzanakos Phys. Rev. Lett. 34, 1047(1975)

5. V. Chaloupka et. al., Phys. Lett. $\underline{61 \mathrm{~B}}$, 487(1976).

6. P. Benkheiri et. al., Phys. Lett. 68 B, 483(1977).

7. J. Bodenkamp et. al., Phys. Lett. 133 B, 275(1983).

8. B.G. Gibbard et. al., Phys. Rev. Lett. 42, 1593(1979).

9. R. Bizzarri et. al., Phys. Rev. D $\underline{6}, 160(1972)$.

10. J. Bensinger et. al., Phys. Rev. D 23, 1417(1983).

11. M.W. Eaton et. al., Phys. Rev. D 29, 805(1984).

12. B.A. Mecking et. al., NIM A503, 513(2003). 